Reprod. Nutr. Dévelop., 1986, 26 (2 B), 523-541.

\title{
Contrôle hormonal du développement et de l'activité de la glande mammaire
}

L. M. HOUDEBINE

Laboratoire de Physiologie de la Lactation

I.N.R.A., 78350 Jouy-en-Josas, France.

La glande mammaire est depuis de nombreuses années l'objet d'études dont une des caractéristiques est certainement la grande diversité. Ceci tient naturellement au fait que : $1^{\circ}$ ) cet organe constitue un remarquable modèle biologique, $\left.2^{\circ}\right)$ qu'il produit une des nourritures essentielles de l'humanité, $3^{\circ}$ ) que $s^{\prime} y$ développent des tumeurs avec une fréquence relativement élevée dans l'espèce humaine.

La glande mammaire doit produire des quantités considérables de protéines, de lipides et de lactose pendant la lactation et rester inactive en dehors de cette période. C'est évidemment ce qui justifie l'existence cyclique du tissu sécréteur qui n'apparaît que pendant la dernière partie de la gestation, ne devient actif qu'après la parturition et disparaît après le sevrage. L'induction de la sécrétion lactée est donc l'aboutissement d'une série de phénomènes qui constituent autant d'amplifications successives : apparition des cellules épithéliales sécrétrices, organisation du tissu en alvéoles et canaux, activation des fonctions spécifiques des cellules sécrétrices (gènes des protéines du lait et des enzymes impliquées dans la synthèse du lactose et des lipides), hypertrophie et polarisation des cellules. Toutes ces transformations, aussi impressionnantes soient-elles, resteraient évidemment inopérantes si les cellules, une fois différenciées, n'étaient pas convenablement alimentées en éléments précurseurs du lait. Le métabolisme maternel se trouve donc réorienté après la parturition pour satisfaire les exigences de la glande mammaire qui va consommer, entre autres, au cours de la lactation, une part considérable du glucose circulant. Tous ces phénomènes sont coordonnés, en grande partie, par des hormones dont les principales ont été définies depuis longtemps. Le rôle réel de certaines d'entre elles reste toutefois incertain et ce d'autant plus qu'il existe des différences notables entre les espèces. II est clair, par ailleurs, que certains des facteurs hormonaux qui gouvernent le développement du tissu sécréteur restent à découvrir. La présente revue se propose de faire le point sur certaines découvertes récentes concernant le développement et l'activation du tissu épithélial sécréteur mammaire. 


\section{Rôle des androgènes.}

L'ébauche mammaire qui se forme au cours de la vie fœtale dérive de l'ectoderme. Il est établi depuis longtemps que chez le fœetus mâle, le tissu mammaire subit une nécrose partielle qui interdit d'emblée tout développement important de la glande chez l'adulte. Ce phénomène est particulièrement marqué chez la souris où la nécrose va jusqu'à faire disparaître les tétons. Ce sont les androgènes produits par les testicules du fœtus mâle qui induisent la nécrose du parenchyme mammaire au $14^{\mathrm{e}}$ jour de la gestation. Une injection d'androgènes à des fœetus femelles conduit à un phénotype mâle, donc à une nécrose du tissu mammaire, tandis que des injections d'acétate de cyproterone (un anti-androgène puissant) à des fœtus mâles conduit à un phénotype femelle. L'action des androgènes ne s'exerce pas directement sur le parenchyme mammaire qui subit la nécrose. Ce sont en effet les cellules du mésenchyme qui possèdent les récepteurs des androgènes. La présence de ces récepteurs nécessite par ailleurs le contact entre les cellules du mésenchyme et celles du parenchyme. Ce sont donc les cellules épithéliales qui permettent aux cellules du mésenchyme de percevoir le message des androgènes et ce sont les cellules du mésenchyme ainsi stimulées qui induisent la nécrose du parenchyme par l'intermédiaire d'un mécanisme qui reste inconnu (Heuberger et al., 1982 ; Wasner et al., 1983).

\section{Rôle des œstrogènes.}

Jusqu'à la puberté, le tissu mammaire ne subit aucune croissance privilégiée. Ce n'est que sous l'influence des stéroïdes ovariens qui augmentent de manière cyclique après la puberté que la glande mammaire commence à se développer. Ce développement reste toutefois extrêmement limité et c'est essentiellement la mise en place d'un réseau de canaux qui a lieu chez la plupart des espèces. Le tissu sécréteur proprement dit n'apparaît que dans le dernier tiers de la gestation. Par ailleurs, il est possible d'induire une production de lait très significative chez des femelles vierges ou taries en leur injectant des œstrogènes pendant un temps relativement bref (Head et al., 1982). Il est d'autre part bien connu qu'un certain nombre de tumeurs mammaires sont œstrogéno-dépendantes. Ces faits expérimentaux indiquent clairement que les oestrogènes orit un rôle clé dans le déclenchement du développement de la glande mammaire. On peut donc considérer que c'est la persistance à un taux élevé de cette hormone au cours de la gestation qui induit l'apparition du tissu sécréteur. II ne faudrait toutefois pas en conclure que les œstrogènes sont des facteurs de croissance pour la glande mammaire. En effet, ces stéroïdes ne stimulent la multiplication des cellules mammaires in vitro que dans le cas de certaines lignées dérivées de tumeurs. Les cellules épithéliales mammaires normales en culture sont toutefois parfaitement sensibles aux œestrogènes qui induisent une accumulation des récepteurs de la progestérone (Edery et al., 1984a). Cette induction ne s'accompagne d'aucune stimulation de la multiplication cellulaire qui dépend de la progestérone et de la prolactine (Edery et al., 1984b). Il est donc clair que les œestrogènes délivrent in vivo un message d'une 
nature totalement inconnue, ce qui permet le développement de la glande mammaire, lui-même assuré par divers facteurs de croissance. Le message œstrogénique n'est d'ailleurs pas strictement obligatoire puisque des injections prolongées de quantités importantes de prolactine à des rates castrées induisent un abondant développement du tissu mammaire sécréteur (Talwalker and Meites, 1961).

Indépendamment de leur intervention dans le déclenchement de la croissance mammaire, les œstrogènes exercent une action décisive pour maintenir le tissu épithélial sensible aux hormones lactogènes. En effet, chez la souris ou la rate vierge mature, une association d'insuline, de prolactine et de cortisol induit in vitro la synthèse de caséine et l'accumulation des ARNm correspondants. Cette induction n'a plus lieu si les animaux ont été préalablement castrés et elle est restaurée si les cestrogènes sont injectés avant le prélèvement du tissu destiné à la culture (Bolander et Topper, 1980 ; Sankaran et al., 1984). Cette perte de sensibilité aux hormones lactogènes en absence d'œstrogènes n'est pas générale mais au contraire sélective puisque l'insuline est toujours capable d'augmenter l'entrée de l'acide $\alpha$-amino butyrique, le cortisol d'induire la NADH-cytochrome c réductase et la prolactine de stimuler la synthèse des ARN totaux (Sankaran et al., 1984). Le manque d'œstrogènes a donc induit une lésion biochimique réversible et sélective dans le tissu mammaire. La nature du message œstrogénique reste totalement inconnu et il ne semble être émis et perçu qu' in vivo.

Des études réalisées chez la souris ont clairement démontré qu'une induction de la synthèse de lait par les hormones en culture organotypique ne pouvait pas être observée tant qu'une multiplication cellulaire n'avait pas eu lieu. Ces faits ont conduit à formuler l'hypothèse qu'une mitose différenciatrice précédait nécessairement l'induction de la synthèse du lait. De telles observations n'ont pas pu être faites chez d'autres espèces (Houdebine et Djiane, 1980). Une étude comparée récente amène à conclure que cette théorie n'est pas généralisable mais qu'au contraire, la souris représente à cet égard une exception qui n'a pas reçu d'explication en termes de mécanisme moléculaire ou cellulaire (Nicholas et al., 1983). Les cellules sont donc en règle générale très tôt sensibles aux hormones lactogènes, l'existence du "lait de sorcière " chez les nouveau-nés vient renforcer cette proposition.

\section{Rôle de la progestérone.}

Au cours du cycle gestation-lactation, le taux de la progestérone plasmatique n'est élevé que pendant la gestation. On peut donc s'attendre à ce que cette hormone favorise le développement de la glande mammaire tout en maintenant son activité à un niveau réduit (Houdebine et al,, 1983). C'est en effet ce que l'on observe puisque la progestérone, inactive seule, favorise les effets des œestrogènes sur la sensibilisation du tissu mammaire aux facteurs de croissances et aux hormones lactogènes (Nicholas et al., 1981; Head et al., 1982). La progestérone stimule par ailleurs la croissance du tissu mammaire in vivo (Assairi et al., 1974) et in vitro (Edery et al., 1984b). La progestérone exerce son effet inhibiteur sur la lactogenèse par au moins deux voies indépendantes : $1^{\circ}$ ) elle inhibe la sécrétion de prolactine par I'hypophyse (Kann et al., 1978), $2^{\circ}$ ) elle atténue fortement les 
effets de la prolactine exogène (Assairi et al., 1974 ; Teyssot et Houdebine, 1980). Il est donc clair que c'est la chute du taux de progestérone en fin de gestation qui constitue un des signaux majeurs du déclenchement de la sécrétion lactée. Le mécanisme d'action de la progestérone reste très mal compris. II est en effet surprenant que cette hormone exerce si franchement son action inhibitrice in vivo et si difficilement in vitro (Teyssot et Houdebine, 1980). Une inhibition a toutefois été observée dans un certain nombre de cas in vitro mais avec des concentrations très élevées d'hormone $(1 \mu \mathrm{g} / \mathrm{ml})$. La signification réelle de telles expériences reste vague et on peut se demander si la progestérone agit véritablement en contrecarrant les effets des glucocorticoïdes dans la mesure où les taux circulants de ces deux stéroïdes sont peu différents tout au long de la gestation (Ganguly et al., 1982). Un fait apparaît toutefois assez clairement : la synthèse $d^{\prime} \alpha$-lactalbumine et partant de lactose est très aisément inhibée, même en culture organotypique, à des concentrations physiologiques de progestérone (Goodman et al., 1983). La signification biologique de ces différences de sensibilité vis-à-vis de la progestérone est incertaine. On peut penser que l'absence de lactose assurée par la progestéronémie élevée tout au long de la gestation interdit en même temps le passage d'eau dans les lumières des alvéoles et réduit ainsi au maximum la sécrétion lactée.

\section{Rôle de I'hormone placentaire lactogène.}

Il est malaisé de définir clairement l'influence réelle des hormones placentaires lactogènes sur la glande mammaire. En effet, certaines espèces en sont dépourvues. D'autre part, chez une espèce comme la vache, l'hormone est présente dans le placenta mais pas dans le sang et une induction de lactation peut parfaitement avoir lieu chez des animaux non gestants (Head et al., 1982). II n'est pas contestable qu'in vivo cette hormone peut remplacer la prolactine pour induire une sécrétion lactée en présence de cortisol (Martal et Djiane, 1977) et lors de cocultures de placenta et de glande mammaire, une sécrétion de lait peut avoir lieu même en présence d'anticorps anti-prolactine (Servely et al., 1983). Par ailleurs, l'hormone placentaire lactogène ne peut pas participer directement et de manière décisive à la montée laiteuse après la parturition puisque cette hormone disparaît rapidement alors que la prolactine est à son plus haut niveau. Un examen comparé de l'activité lactogène de la prolactine ovine et de l'hormone placentaire lactogène ovine chez la lapine et la brebis a révélé que l'hormone d'origine placentaire était nettement plus active chez la lapine que chez la brebis et qu'elle était beaucoup moins active que la prolactine chez la brebis (Servely et al., 1983). L'activité lactogène des hormones placentaires est donc peut-être moins importante qu'on ne l'imaginait. Les hormones placentaires peuvent toutefois contribuer directement ou via la formation de somatomédines à la croissance de la glande mammaire pendant la gestation. Cette proposition est compatible avec le fait que les hormones placentaires lactogènes sont plus apparentées dans leur structure aux hormones de croissance qu'aux prolactines (Niall et al., 1971). Une étude récente réalisée chez la chèvre indique de plus que l'hormone placentaire est, pour la glande mammaire, un facteur de croissance probablement moins effi- 
cace que la prolactine (Forsyth et al., 1985). Les hormones placentaires lactogènes constituent donc vraisemblablement des hormones de secours dont I'utilité est relativement mineure dans des conditions normales mais qui peuvent se révéler bénéfiques dans des situations où la prolactine est anormalement basse.

\section{Rôle de l'hormone de croissance.}

II est admis depuis longtemps que l'hormone de croissance augmente la production laitière chez les ruminants mais pas chez les autres espèces (Peel et al., 1981). Le mécanisme d'action de cette hormone reste très mal connu. Une partie de l'action pourrait s'exercer au niveau de la glande elle-même puisque sous son influence, le flux sanguin mammaire est augmenté (Mepham et al., 1984). Il est vraisemblable qu'une partie importante de l'action résulte d'une réorientation du métabolisme maternel en faveur de la glande mammaire (Bauman et Curie, 1980). La disponibilité de l'hormone en grande quantité ainsi que celle de la somatocrinine va sans aucun doute apporter dans ce domaine un grand nombre d'informations nouvelles dans un proche avenir.

II n'est pas exclu par ailleurs que l'hormone de croissance agisse directement sur la glande mammaire pour en stimuler le développement. Le complexe mammogène optimum in vitro contient en effet cette hormone (Lyons et al., 1958) et la production d'un facteur de croissance spécifique des cellules épithéliales est induite dans les cellules adipeuses mammaires par l'hormone de croissance (Rudland et al., 1984).

\section{Rôle de la prolactine.}

La prolactine joue un rôle essentiel à toutes les étapes du développement et de la différenciation de la glande mammaire chez la plupart des espèces. II n'est pas certain que la prolactine soit le facteur de croissance essentiel de la glande mammaire (Smith et al., 1984) mais elle favorise la mise en place des structures lobulo-alvéolaires in vivo (Talwalker et Meites, 1961) et in vitro (Lyons et al., 1958 ; Djiane et al., 1982).

La prolactine, même si elle n'est que modérément active seule, est le facteur essentiel qui déclenche la synthèse des caséines. Cette induction s'accompagne d'une accumulation des ARNm et d'une accélération de la transcription des gènes correspondants (Guyette et al., 1979 ; Teyssot et Houdebine, 1980). Pour cette raison, le mécanisme d'action de cette hormone a été particulièrement étudié. $\mathrm{Si}$ peu de choses sont encore comprises, certaines étapes de ce mécanisme sont maintenant connues. Le récepteur a été purifié et des anticorps polyclonaux et monoclonaux dirigés contre ce récepteur miment les actions mammogènes et lactogènes de l'hormone lorsqu'ils sont à l'état divalent (Djiane et al., 1981 ; Dusanter-Fourt et al., 1984 ; Houdebine et al., 1985a). Ces faits indiquent clairement que des événements essentiels dans le transfert du message hormonal aux gènes se déroulent au niveau de la membrane périphérique. 
La prolactine exerce une action déterminante au cours de la période qui entoure la parturition. En effet, la suppression transitoire de la prolactine se traduit par une production laitière particulièrement basse (Kann et al., 1978). II semble que le niveau élevé de prolactine qui accompagne la parturition soit indispensable chez toutes les espèces. La montée laiteuse s'accompagne de I'hypertrophie des cellules épithéliales qui est essentielle pour que soit synthétisée et sécrétée une grande quantité de lait et cette profonde transformation de cellules alvéolaires ne peut avoir lieu en absence de prolactine. II est possible de provoquer l'hypertrophie des cellules épithéliales par des injections de prolactine (Assairi et al., 1974), mais pour des raisons encore inconnues, un tel phénomène ne se déroule jamais lorsque le tissu mammaire est stimulé in vitro. La sécrétion lactée est entretenue chez beaucoup d'espèces par une série d'hormones dont la prolactine fait partie. Des injections de bromocriptine qui supprime la sécrétion de prolactine s'accompagnent chez la lapine ou la rate d'un abaissement important de la production laitière. Tel n'est pas le cas chez les ruminants chez lesquels la production laitière est entretenue surtout par l'hormone de croissance (Peel et al., 1981).

\section{Rôle de l'insuline.}

L'insuline n'est généralement pas considérée comme faisant partie du complexe hormonal lactogène. Ceci tient au fait : $1^{\circ}$ ) que l'insulinémie varie peu, $2^{\circ}$ ) qu'elle est plutôt faible pendant la lactation, $3^{\circ}$ ) qu'il est difficile de la faire varier de manière durable sans causer de graves perturbations chez l'animal. Cette hormone est toutefois systématiquement ajoutée aux milieux de culture dans le but supposé de permettre une bonne survie du tissu. II est maintenant clair qu'en absence d'insuline, le tissu mammaire se maintient convenablement en vie mais que la prolactine n'exerce qu'un effet inducteur limité sur la synthèse des caséines, sur l'accumulation de leurs ARNm et sur la transcription de leurs gènes (Devinoy et al., 1978 ; Bolander et al., 1981 ; Kulski et al., 1983 ;Chomczynski et al., 1984 ; Houdebine et al., 1985a). S'il n'est pas douteux que l'insuline exerce un effet amplificateur puissant sur l'action inductrice de la prolactine, son rôle exact reste mal défini. En effet, l'action insulino-mimétique d'anticorps antirécepteurs de l'insuline atteste que l'effet est bien de type insulinique (Nicholas et Topper, 1983). Par opposition, le fait qu'une stimulation intense par l'insuline n'est obtenue qu'à concentration très élevée (Houdebine et al., 1985b) suggère que I'hormone emprunte un récepteur autre que le sien, celui de I'IGF par exemple, pour au moins une partie importante de son action. L'hypothèse la plus simple est donc que l'insuline exerce une action qui lui est propre à des concentrations physiologiques et qu'aux concentrations supraphysiologiques elle mime l'action d'une autre hormone qui reste à définir.

\section{Rôle des glucocorticoïdes.}

Chez toutes les espèces étudiées, les glucocorticoïdes exercent un puissant effet amplificateur sur les actions de la prolactine. Pendant longtemps, la lapine est apparue comme une exception nette puisqu'in vivo et in vitro, la prolactine 
seule était capable d'induire une synthèse de caséine relativement abondante. La souris, le plus souvent utilisée comme modèle expérimental, constituait une référence fondamentalement différente puisqu'in vitro, seule une combinaison comprenant au moins l'insuline, la prolactine et le cortisol présentait une activité lactogène notable. Une comparaison systématique de différentes espèces entre elles (Matusik et Rosen, 1978 ; Nagarah et al., 1981 ; Houdebine et al., 1985b) a fait apparaître la souris plutôt que la lapine comme une exception. Un examen plus attentif a permis de montrer par ailleurs que chez la rate, une dépendance franche vis-à-vis des glucocorticoïdes apparaît dès lors que les animaux sur lesquels est prélevé le tissu mammaire sont surrénalectomisés (Kulski et al., 1983). De même, les cellules de glande mammaire de lapine acquièrent une plus grande dépendance vis-à-vis du cortisol après des cultures cellulaires de longue durée en absence du stéroïde (Servely, 1983). Il existe donc une mémorisation de l'influence des corticoïdes dont le support moléculaire est vraisemblablement le stéroïde lui-même (Bolander et al., 1979). Les principales espèces étudiées sont donc à peu près également sensibles à la prolactine in vitro en absence de glucocorticoïdes, la souris constituant une exception. La lapine demeure in vivo une espèce particulièrement sensible au stimulus prolactinique.

Il est bon de remarquer que toutes les protéines du lait ne sont pas soumises à la même régulation hormonale. En effet, l'induction de la synthèse de l' $\alpha$ lactalbumine est diminuée par les glucocorticoïdes chez la souris (Ono et Oka, $1980 \mathrm{a}, \mathrm{b})$ tandis que celle d'une protéine majeure du lait de souris et de rate, la WAP (Whey acidic protein) est particulièrement stimulée par les glucocorticoïdes (Hobbs et al., 1982). L'effet inhibiteur des glucocorticoïdes sur l'induction de la synthèse d' $\alpha$-lactalbumine est levé par les prostaglandines $E_{2}$ et $F_{2} \alpha$ (Terrada et al., 1982, 1983) qui sont sans effet sur la synthèse des caséines. Le mécanisme d'action des glucocorticoïdes sur la synthèse des caséines est inconnu. Chez la souris (Ganguly et al., 1979) et la lapine (Teyssot et Houdebine, 1981), ils stimulent la transcription des gènes des caséines, tandis que chez la rate ils pourraient plutôt augmenter la stabilité des produits de transcription (Guyette et al., 1979).

La spermidine a été proposée comme médiateur intracellulaire des glucocorticoïdes (Oka et Perry, 1976). Ceci ne semble pas être le cas chez la lapine (Houdebine et al., 1978). Une étude comparée n'a pas vraiment résolu cette question faute de contrôles appropriés (Bolander et Topper, 1979).

Les glucocorticoïdes inhibent l'activateur du plasminogène (Ossowski et al., 1979) et partant la collagénase mammaire (Salomon et al., 1981). Les glucocorticoïdes jouent donc un rôle essentiel pour stabiliser le collagène que l'on sait important pour que la prolactine perçoive le message prolactinique.

\section{Rôle de l'hormone thyroïdienne.}

L'hormone thyroïdienne exerce une action amplificatrice limitée lors de l'induction de la synthèse de l' $\alpha$-lactalbumine in vitro (Vonderhaar, 1977 ; Terrada et Oka, 1982 ; Bhattacharjee et Vonderhaar, 1984). L'hormone tyroïdienne n'a qu'une activité faible ou nulle sur l'expression des gènes des caséines. 


\section{Rôle de I'EGF.}

Le facteur de croissance de l'épiderme (EGF) stimule la multiplication des cellules épithéliales mammaires (Martel et Houdebine, 1982 ; Taketami et Oka, $1983 a, b)$. L'EGF exerce en même temps un effet inhibiteur sur l'induction de la synthèse de I' $\alpha$-lactalbumine et des caséines (Taketami et Oka, 1983b ; Sankaran et Topper, 1984). L'EGF dont la concentration sanguine est élevée au cours de la gestation (Anges, 1973) pourrait contribuer, comme la progestérone, à favoriser le développement de la glande mammaire tout en limitant son activité. L'action de I'EGF a été comparée à celle des esters de phorbols (Taketami et Oka, 1983c). La signification exacte de ces faits expérimentaux reste incertaine. Il est bon de noter que I'EGF stimule la synthèse de collagène (Salomon et al., 1981). Cette action pourrait représenter une contribution majeure à l'action mitogène de I'EGF.

\section{Les facteurs de croissance de la glande mammaire.}

II est raisonnable de considérer que les deux facteurs essentiels qui limitent la production de lait chez une femelle sont : $1^{\circ}$ ) le nombre de cellules épithéliales mammaires, $2^{\circ}$ ) la capacité qu'a l'organisme maternel d'orienter son métabolisme en faveur de la glande mammaire. L'identification et l'étude du mode d'action des facteurs qui contrôlent le développement de la glande mammaire sont donc essentielles. Une telle approche ne peut par ailleurs qu'aider à comprendre les phénomènes qui conduisent à l'apparition des tumeurs mammaires. Il est bon de noter tout d'abord que pour aucun type cellulaire, la totalité des facteurs qui interviennent aux différentes étapes du cycle cellulaire n'a été clairement définie (Campisi et Pardee, 1984). Une complexité supplémentaire vient se surajouter dans le cas de la glande mammaire qui est composée de plusieurs types cellulaires dont les interactions sont mal connues. Parmi les divers facteurs de croissance, il convient de distinguer : $1^{\circ}$ ) des facteurs hormonaux classiques véhiculés par la voie sanguine, $2^{\circ}$ ) des facteurs de croissance également présents dans le sérum mais dont la nature exacte et la spécificité $d^{\prime}$ action reste à définir, $3^{\circ}$ ) des facteurs de croissance produits en très petite quantité et agissant in situ. Dans un assez grand nombre de tissus, les hormones qui contrôlent la différenciation sont aussi responsables de la multiplication de leurs cellules cibles. La glande mammaire n'échappe pas à cette règle. Ainsi, la prolactine est un des facteurs de croissance de la glande mammaire. Il est bon de noter toutefois que si son action est assez nette in vivo, elle est beaucoup plus modérée in vitro où elle n'agit qu'en synergie avec l'insuline à haute concentration, les glucocorticoïdes et la progestérone (Djiane et al., 1982 ; Martel et Houdebine, 1982 ; Edery et al., 1984a, Smith et al., 1984). Ces différences entre les actions de la prolactine in vivo et in vitro ne sont pas aisément explicables. Une hypothèse formulée récemment suggère que la prolactine in vivo induit la formation d'un facteur de croissance qui accède à la glande mammaire par voie sanguine. Ce facteur qui a reçu le nom de synlactine n'a pas encore été défini avec certitude (Anderson et al., 1983, 1984 ; Nicoll et al., 1985).

Un facteur présent dans l'hypophyse, inductible par la prolactine et stimulant 
spécifiquement la multiplication des cellules mammaires de type épithélial est un bon candidat pour être la synlactine (lkeda et al., 1984 ; Smith et al., 1984). La prolactine n'est de toute évidence pas le seul facteur mitogène essentiel pour la glande mammaire (Smith et al., 1984). Cette constatation est probablement assez peu surprenante. En effet, la prolactine qui est impliquée dans le contrôle d'un grand nombre de fonctions physiologiques subit, en dehors de la gestation, de très nombreuses fluctuations qui ne sont pas accompagnées d'un développement significatif de la glande mammaire. Cette proposition reste probablement acceptable même si I'on considère que la prolactine ne peut agir efficacement qu'après la montée des œstrogènes au cours de la gestation. Dans cet ordre d'idées, il apparaît que les cellules du stroma (fibroblastes, adipocytes) sont stimulées surtout par le FGF (facteur de croissance des fibroblastes), tandis que l'EGF (facteur de croissance de l'épiderme) présente peu de spécificité vis-à-vis du type cellulaire (Smith et al., 1984). Il semble par ailleurs que les différents tissus qui composent la glande mammaire sont maintenus ou développés préférentiellement en fonction des hormones présentes. Ainsi, les gros canaux primaires se maintiennent même en absence d'hormones tandis que les canaux secondaires et tertiaires ont besoin d'insuline et que les bourgeons terminaux disparaissent en l'absence d'insuline, de glucocorticoïdes et de prolactine (Dubois et Elias, 1984).

II est admis depuis longtemps que les œstrogènes favorisent le déclenchement du développement sans être eux-mêmes des facteurs de croissance proprement dits. II a été proposé que ces stéroïdes induisent la formation de facteurs de croissance dans l'hypophyse, l'utérus et le rein. Ces facteurs nommés œstromédines (Ikeda et Sirbasku, 1984) ne sont peut-être pas des facteurs de croissance pour la glande mammaire. En effet, il n'existe aucun fait expérimental qui démontre clairement que les cestromédines migrent vers la glande mammaire. D'autre part, les œstromédines ne semblent pas stimuler réellement les cellules mammaires (Smith et al., 1984). Les œestromédines sont donc vraisemblablement des facteurs de croissance induits par les œstrogènes mais sans rapport avec la glande mammaire.

II est clair que des résultats réellement convaincants concernant les facteurs de croissance de la glande mammaire ne peuvent être obtenus que si l'on distingue clairement les différents types de cellules et leurs interactions. C'est la démarche qu'a entreprise depuis plusieurs années le groupe de $P$. Rudland. A partir de tumeurs mammaires induites chez le rat par le DMBA (dimethyl benzanthracène), des clones cellulaires correspondants à des cellules de type épithélial ou fibroblastique ont été obtenus (Bennett et al., 1978 ; Rudland et al., 1979). En fonction des conditions de culture, les cellules épithéliales peuvent se transformer en cellules myoépithéliales qui ont pu être clonées elles-mêmes ainsi. Cette transformation s'accompagne de l'induction de protéines spécifiques (Barraclough et al., 1982, 1984a). Le gène d'une de ces protéines a été caractérisé (Barraclough et al., 1984b). De même, les cellules épithéliales clonées, sous l'influence des hormones lactogènes et du DMSO (dimethyl sulfoxide) ou de l'acide rétinoïque, sont induites reversiblement pour synthétiser des protéines du lait et des lipides (Rudland et al., 1983). II est possible que l'orientation des cellules épithéliales vers l'état différencié explique l'effet anti-tumoral de l'acide rétinoïque. 
En présence de sérum de cheval ou d'hormone de croissance, les fibroblastes mammaires clonés se transforment en cellules de type adipocyte. Cette transformation s'accompagne de la sécrétion d'un facteur de croissance spécifique des cellules épithéliales. Ce facteur a été identifié comme étant une prostaglandine, la $\mathrm{PGE}_{2}$ (Rudland et al., 1984). Il est intéressant de noter que la souche de fibroblastes non mammaires 3 T3 que l'on sait être les précurseurs de préadipocytes, sécrète un facteur de même type.

Ces travaux remarquables font apparaître des filiations et des interactions inconnues jusqu'alors entre les différents types cellulaires qui composent la glande mammaire. Ils révèlent l'existence de facteurs de croissance spécifique et un rôle peut-être nouveau pour l'hormone de croissance.

D'autres facteurs de croissance favorisant la croissance des cellules mammaires ont été identifiés. Citons pêle-mêle : la phosphoéthanolamine (Kano-Sueoka et al., 1979a), l'ion lithium (Tomooka et al., 1983), des facteurs protéiques extraits du sérum (Porzig et Stockdale, 1982), de I'hypophyse (Kano-Sueoka et al., $1979 b$ ) ou de la glande mammaire (Swanson et Moore, 1979). Mentionnons également l'existence de facteurs de croissance présents dans le lait ou le colostrum (Sinha et Yunis, 1983 ; Brown et Blakeley, 1984 ; Shing et Klagsburn, 1984). Deux de ces facteurs ont été identifiés comme étant l'un l'EGF (Carpenter, 1980) et l'autre l'IGF (ou somatomédine C) (Baxter et al., 1984). La présence de ces facteurs en relative abondance dans le lait ou le colostrum a une signification incertaine. Il reste à déterminer si la cible de ces facteurs est la glande mammaire de la mère ou plutôt les tissus digestifs du nouveau-né.

Le cAMP est généralement considéré comme un des médiateurs intracellulaires de la croissance des cellules mammaires. II est remarquable que l'inverse semble être vrai pour les cellules mammaires tumorales dont la multiplication est freinée par le cAMP (Fentiman et al., 1984).

Une mention particulière doit être faite sur certains travaux récents concernant les relations entre la croissance et la différenciation des cellules épithéliales. Des cellules épithéliales mammaires isolées sont à peu près insensibles aux stimuli de croissance que sont l'insuline et le cortisol en présence de sérum. A l'état associé, et dans des conditions hormonales similaires, les mêmes cellules formant des " unités de croissance » se multiplient. Mais cette multiplication cesse spontanément après 3 ou 4 divisions indépendamment du degré de confluence des cellules. Les cellules peuvent alors se différencier et synthétiser du lait. Cette inhibition de croissance nommée " inhibition de renouvellement ", par opposition à l'inhibition de contact que l'on observe classiquement dans les cellules tumorales, pourrait être le signal qui permet la différenciation (Mc Grath et Soule, 1983). II est évidemment tentant d'imaginer que ces expériences reproduisent ce qui se passe in vivo lors de la formation et de l'activation des alvéoles.

Dans le même ordre d'idées, il semble que l'ion calcium, à faible concentration $(<0,06 \mathrm{mM})$, favorise la croissance des cellules épithéliales, mammaires, aux dépends de leur différenciation, alors qu'à concentration généralement considérée comme physiologique, les complexes hormonaux lactogènes classiques induisent plutôt la différenciation que la multiplication des mêmes cellules (Mc Grath et Soule, 1984). 


\section{Rôle de la matrice extracellulaire.}

Des fragments de glande mammaire prélevés chez des animaux gestants sont parfaitement sensibles aux hormones lactogènes. Des petits agrégats de cellules résultant d'une digestion partielle du collagène mammaire sont encore bien actifs lorsqu'ils sont cultivés sur un support plastique classique. Ces cellules complètement isolées et cultivées dans les mêmes conditions sont devenues beaucoup moins sensibles aux hormones. Après être resté sans explication pendant longtemps, ce phénomène est maintenant assez bien compris. L'interaction entre la matrice extracellulaire mammaire et les cellules est indispensable pour que le message hormonal soit perçu de manière intense. La matrice extracellulaire mammaire est composée essentiellement de collagène I, III et IV, de glycoprotéines et de protéoglycanes. Le collagène IV, la fibrine et la laminine sont synthétisés et sécrétés par les cellules myoépithéliales tandis que les fibroblastes sont à l'origine des collagènes I et III. La matrice extracellulaire se forme normalement au contact du parenchyme mammaire et du tissu conjonctif. Des cellules épithéliales mammaires peuvent être cultivées sur une fraction brute de collagène préparé à partir de tendon de queue de rat et composée essentiellement de collagène de type 1. Dans ces conditions, elles se multiplient, s'organisent en trois dimensions sous forme de structures ressemblant à celles que l'on observe dans la glande mammaire et elles se différencient en sécrétant des quantités substantielles de caséines (Emerman et al., 1977 ; Burwen et Pitelka, 1980 ; Shannon et Pitelka, 1981 ; Edery et al., 1984a, b ; Lee et al., 1984). Il est intéressant de constater que les cellules clonées cuboïdales de type épithélial s'organisent dans le collagène en des formes qui ressemblent à des canaux sans bourgeon terminal, tandis que les cellules myoépithéliales ne forment que des pointes composées de cellules aliongées et que les cellules fibroblastiques migrent dans le collagène sans donner lieu à une organisation multicellulaire (Ormerod et Rudland, 1982). Le collagène n'est opérant que s'il est flottant et il perd toute son efficacité lorsqu'il est rigidifié par un traitement au glutaraldéhyde. Un des rôles du collagène | ajouté sous forme de gel et sur lequel sont ensemencées les cellules consiste à stabiliser les protéoglycanes. Ces molécules sont en effet synthétisées à un taux constant par les cellules, qu'elles adhèrent au plastique ou au collagène (David et Bernfield, 1981). La synthèse des collagènes de type I et III semble être indispensable pour que le message hormonal des hormones lactogènes soit normalement perçu par les cellules épithéliales. En effet, un analogue de la proline, l'acide L-azétidine-2 carboxylique qui inhibe la synthèse de ces collagènes prévient presque totalement l'induction de la synthèse des protéines du lait (Vonderhaar et al., 1978). De même, l'inhibition de la synthèse du collagène de type IV par la cis-hydroxy proline s'oppose à la prolifération des cellules épithéliales (Wicha et al., 1980). Les éléments autres que le collagène sont également importants et une matrice mammaire totale peut remplacer le collagène I (Wicha et al., 1982 ; Wilde et al., 1984). Certains éléments du contrôle de la formation de la matrice extracellulaire sont connus. Ainsi, I'EGF induit spécifiquement la synthèse du collagène III. Cet effet de I'EGF pourrait être une partie essentielle de son mécanisme d'action sur la multiplication des cellules mammaires (Salomon et al., 1981). De même, les glucocorti- 
coïdes inhibent la formation de l'activateur du plasminogène (Ossowski et al., 1979) et, en cascade, l'activation de la collagénase (Salomon et al., 1981) ; ces effets pourraient rendre compte du fait qu'ils font partie du complexe hormonal lactogène et mammogène. Les éléments qui composent la matrice contrôlent euxmêmes la formation de la matrice. Ainsi, des cellules épithéliales cultivées sur support plastique sécrètent des glucosaminoglycanes dans le milieu de culture. Ces molécules ne sont intégrées sous forme de matrice qu'en présence de collagène de préférence flottant (Parry et al., 1985).

Les interactions entre les divers éléments qui composent la glande mammaire sont donc particulièrement complexes puisqu'il faut considérer les différents types cellulaires et les composants de la matrice extracellulaire. A cet égard, il est intéressant de signaler que si les cellules épithéliales isolées cultivées dans du collagène reforment des structures de type canaliculaire, les bourgeons terminaux qui sont les précurseurs directs des alvéoles, n'apparaissent que lorsque les extrémités des canaux ainsi néo-formés se rapprochent d'adipocytes qui doivent délivrer un message de nature totalement inconnue (Daniel et al., 1984).

\section{Conclusions.}

Les faits expérimentaux rapportés ici rendent compte des progrès accomplis ces dernières années dans la compréhension des phénomènes qui accompagnent la différenciation de la cellule épithéliale mammaire. Un des faits les plus saillants est sans aucun doute le clonage des différents types cellulaires qui permet désormais de définir de manière claire leur rôle exact, les éléments qui constituent leur activité et leurs interactions avec la matrice extracellulaire. II n'est pas hasardeux de considérer que des progrès importants vont encore être réalisés rapidement dans ce domaine. L'étude des divers types cellulaires se fait également par d'autres critères plus fins qui reposent sur la mise en évidence de marqueurs cellulaires de surface ou de marqueurs intracellulaires (Dulbecco, 1982 ; Dulbecco et al., 1984). De tels marqueurs, lorsqu'ils auront été identifiés et caractérisés de manière plus approfondie, permettront de distinguer les différents types cellulaires et leur filiation, mais aussi leur état de différenciation et leur localisation à l'intérieur du tissu au cours du développement. Les marqueurs de surface sont non seulement la preuve de l'identité d'une cellule, ce sont aussi les éléments qui sont impliqués dans les interactions cellules-cellules et cellules-matrice. Ce sont donc eux qui doivent présider à la formation du tissu mammaire sécréteur. Des relations spatiales ont ainsi pu être établies entre certains de ces antigènes et les éléments de la matrice extracellulaire dans les cellules normales (Monaghan et al., 1983) et tumorales (Terranova et al., 1983). De tels marqueurs peuvent éventuellement être utilisés pour le dépistage de tumeurs (Ceriani et al., 1982 ; Sarkar et al., 1984).

Des progrès considérables ont également été accomplis dans la connaissance de la matrice extracellulaire. Les connaissances acquises trouvent rapidement leur application puisqu'il a pu être démontré que la synthèse de collagène IV dans les tumeurs n'est plus sous la dépendance de l'EGF mais d'un facteur sécrété par les adénocarcinomes eux-mêmes (Bano et al., 1983). De même, certaines cellules tumorales ne perçoivent plus le message du collagène I et elles sont devenues 
incapables de stabiliser les protéoglycanes (David et Bernfield, 1982 ; David et Van den Berghe, 1983).

Les mécanismes intracellulaires qui accompagnent la différenciation restent mal compris. Des comparaisons systématiques entre espèces ont permis de définir avec plus de précision les différences réelles en ce qui concerne les dépendances hormonales et par le fait même ce que toutes ces espèces ont en commun. De grandes incertitudes demeurent quant au rôle réel entre autres de l'hormone de croissance et de l'insuline. Des progrès considérables ont été accomplis dans la connaissance du récepteur de la prolactine qui a pu être caractérisé et isolé. Des anticorps polyclonaux puis monoclonaux (Djiane et al., 1981 ; Houdebine et al., 1985b) qui ont des activités prolactino-mimétiques ont pu être obtenus. Ceci met en évidence le rôle essentiel du récepteur et cet outil permettra de mieux comprendre l'ensemble des événements qui président au transfert de l'information hormonale dans la cellule.

Un progrès assez net a également été réalisé dans la connaissance de la structure et la régulation des gènes cibles de la prolactine: caséines, $\alpha$ lactalbumine, WAP, $\beta$-lactoglobuline (Blackburn et al., 1982 ; Dandekar et al., 1982 ; Gupta et al., 1982 ; Hennighausen et al., 1982a, b ; Horn et al., 1983 ; YuLee et Rosen, 1983 ; Nakhasi et al., 1984 ; Qasba et Safaya, 1984 ; Houdebine et al., 1985b). Ces travaux ont permis de démontrer que dans les cellules tumorales mammaires les gènes des caséines qui ne s'expriment pas ou très peu sont hyperméthylés (Johnson et al., 1983). Peu d'éléments concernant les relations entre la structure fine des gènes et la régulation de leur expression ont jusqu'à maintenant été mis à jour. II n'est pas hasardeux d'affirmer que de telles informations seront disponibles dans un avenir prochain.

Les différentes voies qui sont suivies dans l'étude de la glande font apparaître la grande complexité des phénomènes qui s'y déroulent et la difficulté qu'il y a à les intégrer dans des schémas simples. Ceci est particulièrement vrai en ce qui concerne la membrane qui est le lieu de perception, de transformation, d'intégration et de transmission de la plupart des messages que reçoit la cellule. Les réponses aux questions que l'on se pose ne seront évidemment données que par une approche multidisciplinaire.

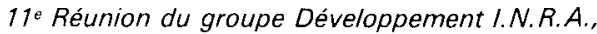
Montpellier, 22-24 mai 1985.

\section{Références}

ANGES I. G., 1973. Serum concentrations of epidermal growth factor in human pregnancy. Am. J. Obstet. Gynecol., 115, 357-362.

ANDERSON T. R., PITTS D. S., NICOLL C. S., 1983. Prolactin's mitogenic action on pigeon crop-sac mucosal epithelium involves direct and indirect mechanisms. Gen. comp. Endocr., 54, $236-246$.

ANDERSON T. R., RODRIGUEZ NICOLL C. S., 1984. The synlactin hypothesis: prolactin's mitogenic action may involve synergism with a somatomedin-like molecule. In Insulin-like growth factors/somatomedins, pp. 71-78. Édité par Walter de Gruyter \& Co, Berlin-New York. 
ASSAIRI L., DELOUIS C., GAYE P., HOUdEBINE L. M., OLLIVIER-BOUSQUeT M., 1974. DENAMUR R. Inhibition by progesterone of the lactogenic effect of prolactin in pseudopregnant rabbit. Biochem. J., 144, 245-252.

BANO M., ZWIEBEL J. A., SALOMON D. S., KIDWELL W. R., 1983. Detection and partial characterization of collagen synthesis stimulating activities in rat mammary adenocarcinomas. J. Biol. Chem., 258, 2729-2735.

BARRACLOUGH R., DAWSON K. J., RUDLAND P. S., 1982. Control of protein synthesis in cuboidal rat mammary epithelial cells in culture. Eur. J. Biochem., 129, 335-341.

BARRACLOUGH R., KIMBEL R., RUDLAND P. S., 1984a. Increased, abundance of a normal cell mRNA sequence accompains the conversion of rat mammary cuboidal epithelial cells to elongated myoepithelial-like cells in culture. Nucleic Acids Res., 12, 8097-8114.

BARRACLOUGH R., DAWSON K. J., RUDLAND P. S., 1984b. Elongated cells derived from rat mammary cuboidal epithelial cell lines resemble cultured mesenchymal cells in their pattern of protein synthesis. Biochim. biophys. Res. Comm., 120, 351-358.

BAUMAN D. E., CURIE W. B., 1980. Partitioning of nutrients during pregnancy and lactation: a review of mechanisms involving homeostasis and homeorhesis. J. Dairy Sci., 63, 1514 1529.

BAXTER R. C., ZALTSMAN Z., TURME J. R., 1984. Immunoreactive somatomedin-c/insulinlike growth factor and its binding protein in human milk. J. clin. Endocrinol. Metab., 58, 955 961.

BENNETT D. C., PEACHEY L. A., DURBIN H. D., RUDLAND P. S., 1978. A possible mammary stern cell line. Cell, 15, 283-298.

BHATTACHARJEE M., VONDERHAAR B. K., 1984. Thyroid hormones enhance the synthesis and secretion of $\alpha$-lactalbumin by mouse mammary tissue in vitro. Endocrinology, 115, 1070-1077.

BLACKBURN D. E., HOBBS A. A., ROSEN J. M., 1982. Rat $\beta$-casein : sequence analysis and evolutionary comparisons. Nucleic Acids Res., 10, 2295-2307.

BOLANDER F. F., TOPPER Y. J., 1979. Relationship between spermidine, glucocorticoid and milk proteins in different mammalian species. Biochem. biophys. Res. Comm., 90, 1131-1135.

BOLANDER F. F., NICHOLAS K. R., TOPPER Y. J., 1979. Retention of glucocorticoid by isolated mammary tissue may complinate interpretation of results from in vitro experiments. Biochem. biophys. Res. Comm., 91, 247-252.

BOLANDER F. F., TOPPER Y. J., 1980. Loss of differentiative potential of the mammary gland in ovariectomized mice : prevention and reversibility of the defect. Endocrinology, 107, 12811285.

BOLANDER F. F., NICHOLAS K. R., VANWYK J. J., TOPPER Y. J., 1981. Insulin is essential for accumulation of casein mRNA in mouse mammary epithelial cells. Proc. nat. Acad. Sci. USA, 78, 5682-5684.

BROWN K. D., BLAKELEY D. M., 1984. Partial purification and characterization of a growth factor present in goat's colostrum. Biochem. J., 219, 609-617.

BURWEN S. J., PITELKA D. R., 1980. Secretory function of lactating mouse mammary epithelial cells cultured on collagen gels. Exp. Cell Res., 126, 249-262.

CAMPISI J., PARDEE A. B., 1984. Post transcriptional control of the onset of DNA synthesis by an insulin-like growth factor. Mol. cell. Biol., 4, 1807-1814.

CARPENTER G., 1980. Epidermal growth factor is a major growth promoting agent in human milk. Science, 210, 198-199.

CERIANI R. L., SASAKI M., SUSSMAN H., WARA W. M., BLANK E. W., 1982. Cirvulating human mammary epithelial antigens in breast cancer. Proc. nat. Acad. Sci. USA, 79, 5420-5424.

CHOMCZYNSKI P., QASBA P., TOPPER Y. J., 1984. Essential role of insulin in transcription of the rat 25,000 molecular weight casein gene. Science, 226, 1326-1328.

DANDEKAR A. M., ROBINSON E. A., APPELLA E., QASBA P. K., 1982. Complete sequence analysis of cDNA clones encoding rat whey phosphoprotein : homology to a protease inhibitor. Proc. nat. Acad. Sci. USA, 79, 3879-3991.

DANIEL C. H., BERGER J. J., STRICKLAND P., GARCIA R., 1984. Similar growth pattern of mouse mammary epithelium cultivated in collagen matrix in vivo and in vitro. Dev. Biol., 104, 57-64. 
DAVID G., BERNFIELD M., 1981. Type I collagen reduces the degradation of basal lamina proteoglycan by mammary epithelial cells. J. Cell Biol., 91, 281-286.

DAVID G., BERNFIELD M., 1982. Defective basal lamina formation by transformed mammary epithelial cells : a reduced effect of collagen on basal lamina (heparan sulfate-rich) proteoglycan degradation. J. cell. Physiol., 110, 56-62.

DAVID G., VAN DEN BERGHE H., 1983. Transformed mouse mammary epithelial cells synthesize undersulfated basement membrane proteoglycan. J. biol. Chem., 258, 7338-7344.

DEVINOY E., HOUDEBINE L. M., DELOUIS C., 1978. Role of prolactin and glucocorticoids in the expression of casein genes in rabbit mammary gland oigan culture. Biochim. biophys. Acta, 517, 360-366.

DJIANE J., HOUDEBINE L. M., KELLY P. A., 1981. Prolactin-like activity of anti-prolactin receptor antibodies on casein and DNA synthesis in the mammary gland. Proc. nat. Acad. Sci. USA, 78, 7445-7448.

DJIANE J., HOUDEBINE L. M., KELLY P. A., 1982. Correlation between prolactin-receptor interaction, down-regulation of receptors, and stimulation of casein and deoxyribonucleic acid biosynthesis in rabbit mammary gland explants. Endocrinology, 110, 791-795.

DUBOIS M., ELIAS J. J., 1984. Subpopulations of cells in immature mouse mammary gland as detected by proliferative response to hormones in organ culture. Dev. Biol, 106, 7075.

DULBECCO R., 1982. Immunological markers in the study of development and oncogenesis on the rat mammary gland. J. cell. Physiol., 2, 19-22.

DULBECCO R., ALLEN W. R., BOWMAN M., 1984. Lumen formation and redistribution of inframembranous proteins during differentiation of ducts in the rat mammary gland. Proc. nat. Acad. Sci. USA, 81, 5763-5766.

DUSANTER-FOURT I., DJIANE J., KELLY P. A., HOUDEBINE L. M., TEYSSOT B., 1984 Differential biological activities between mono and bivalent fragments of anti-prolactin receptor antibodies. Endocrinology, 114, 1021-1027.

EDERY M., Mc GRATH M., LARSON L., NANDI S., 1984a. Correlation between in vitro growth and regulation of estrogen and progesterone receptors in rat mammary epithelial cells. Endocrinology, 115, 1661-1697.

EDERY M., IMAGAWA W., LARSON L., NANDI S., 1984b. Regulation of estrogen and progesterone receptor level in mouse mammary epithelial cells grown in serum-free collagen gel cultures. Endocrinology, 116, 105-112.

EMERMAN J. T., ENAMI I., PITELKA D. R., NANDI S., 1977. Hormonal effects on intracellular and secreted casein in cultures of mouse mammary epithelial cells on floating collagen membranes. Proc. nat. Acad. Sci. USA, 74, 4466-4470.

FENTIMAN I. S., DUHIG T., GRIFFITHS A. B., TAYLOR-PAPADIMITRIOU J., 1984. Cyclic AMP inhibits the growth of human breast cancer cells in defined medium. Mol. Biol. Med., 2, 8188.

FORSYTH 1. A., RYATT J. C., ILEVY S., 1985. Hormone concentrations, mammary development and milk yield in goats given long-term bromocryptine treatment in pregnancy. $J$. Endocr., 104, 77-85.

GANGULY R., MEHTA N. M., GANGULY N., BANERJEE M. R., 1979. Glucocorticoid modulation of casein gene transcription in mouse mammary gland. Proc. nat. Acad. Sci. USA, 76 , 6466-6470.

GANGULY R. N., MAJUMDER P. K., GANGULY N., BANERJEE M. R., 1982. The mechanism of progesterone glucocorticoid interaction in regulation of casein gene expression. J. biol. Chem., 257, 2182-2187.

GOODMAN G. T., AKERS M., FRIDERICI K. H., TUCKER H. A., 1983. Hormonal regulation of $\alpha$-lactalbumin secretion from bovine mammary tissue cultured in vitro. Endocrinology, 112. 1324-1330.

GUPTA P., ROSEN J. M., D'EUSTACHIO P., RUDDLE F. H., 1982. Localization of the casein gene family to a single mouse chromosome. J. Cell Biol., 93, 199-204.

GUYETTE W. A., MATUSIK R. J., ROSEN J. M., 1979. Prolactin-mediated transcriptional and post-transcriptional control of casein gene expression. Cell, 17, 1013-1023.

HEAD H. H., DELOUIS C., FĖVRE J., KANN G., TERQUI M., DJIANE J., 1982. Hormone leveis in plasma of ewes induced into lactation. Reprod. Nutr. Dévelop. 22, 641-650. 
HENNIGHAUSEN L. G., SIPPEL A. E., HOBBS A. A., ROSEN J. M., 1982a. Comparative sequence analysis of the mRNAs coding for mouse and rat whey protein. Nucleic Acids Res., 10, 3733-3744.

HENNIGHAUSEN L. G., STEUDLE A., SIPPEL A. E., 1982b. Nucleotide sequence of cloned CDNA for mouse $\epsilon$-casein. Eur. J. Biochem., 126, 569-572.

HEUBERGER B., FITZKA I., WASNER G., KRATOCHWIL K., 1982. Induction of androgen receptor formation by epithelium-mesenchyme interaction in embryonic mouse mammary gland. Proc. nat. Acad. Sci. USA, 79, 2957-2961.

HOBBS A. A., RICHARDS D. A., KESSLER D. J., ROSEN J. M., 1982. Complex hormonal regulation of rat casein gene expression. J. biol. Chem., 257, 3598-3605.

HORN T. M., SODROSKI J., QASBA P. K., 1983. Protein-coding capacities of polyadenylated RNA from normal and neoplastic rat mammary tissues. Cancer Res., 43, 1819-1826.

HOUDEBINE L. M., DEVINOY E., DELOUIS C., 1978. Role of spermidine on casein gene expression in the rabbit. Biochimie, 60, 735-741.

HOUDEBINE L. M., DJIANE J., 1980. Effects of lysosomotropic agents and microfilaments and microtubule disrupting drugs on the activation of casein gene expression by prolactin in the mammary gland. Mol. cell. Endocr., 17, 1-15.

HOUDEBINE L. M., TEYSSOT B., DEVINOY E., OLLIVIER-BOUSOUET M., DJIANE J., KELLY P. A., DELOUIS C., KANN G., FEVRE J., 1983. Role of progesterone in the development and the activity of the mammary gland, 297-319. In C. W., BARDIN, E. MILGROM, P. MAUVAIS-JARVIS, Progesterone and progestins. Raven Press.

HOUDEBINE L. M., DJIANE J., KELLY P. A., KATOH M., DUSANTER-FOURT I., MARTEL P., 1985a. The mechanism of action of prolactin on casein gene expression, 203-206. Proc. 7th int. Congr. Endocr. Elsevier Sci. Publ.

HOUDEBINE L. M., DJIANE J., DUSANTER-FOURT I., MARTEL P., KELLY P. A., DEVINOY E., SERVELY J. L., 1985b. The mechanism of action of the hormones controlling the mammary gland activity. J. Dairy Science, 68, 489-500.

IKEDA T., DANIELPOUR D., SIRBASKU D. A., 1984. Characterization of a sheep pituitary derived-growth factor for rat and human mammary tumors. J. cell. Biochem., 25, 213-229.

IKEDA T., SIRBASKU D. A., 1984. Purification and properties of a mammary uterine-pituitary tumor cell growth factor from pregnant sheep uterus. J. biol. Chem., 259, 4049-4064.

JOHNSON M. L., LEVY J., SUPOWIT S. C., YU-LEE L. Y., ROSEN J. M., 1983. Tissue - and cell - specific casein gene expression. Relationship to site-specific DNA methylation. J. biol. Chem., 258, 10805-10811.

KANN G., CARPENTIER M. C., FEVRE J., MARTINET J., MAUBON M., MEUSNIER C., PALY J., VERMEIRE N., 1978. Lactation and prolactin in sheep, role of prolactin in initiation of milk secretion, 201-212. C. ROBYN, M. HARTER, Progress in prolactin physiology and pathology. Elsevier North Holland, Biomed. Press.

KANO-SUEOKA T., COHEN D. M., YAMAIZUMI Z., MISHIMURA S., MORI M., FUJIKI H., 1979a. Phosphoethanolamine as a growth factor of a mammary carcinoma cell liver of rat. Proc. nat. Acad. Sci. USA, 76, 5741-5744.

KANO-SUEOKA T., ERRICK J. E., COHEN D. M., 1979b. Effects of hormones and a novel mammary growth factor on a rat mammary carcinoma in culture. Cold Spring Harbor Conferences on Cell Proliferation, 6, 499-512.

KULSKI J. K., NICHOLAS K. R., TOPPER Y. J., QASBA P., 1983. Essentiality of insulin and prolactin for accumulation of rat casein mRNAs. Biochem. biophys. Res. Comm., 116, 994999.

KULSKI J. K., TOPPER Y. J., CHOMCZYNSKI P., QASBA P., 1983. An essential role for glucocorticoid in casein gene expression in rat mammary explants. Biochem. biophys. Res. Comm., 114, 380-387.

LEE E. Y. H., PARRY G., BISSELL M. J., 1984. Modulation of secreted proteins of mouse mammary epithelial cells by the collagenous substrata. J. Cell Biol., 98, 146-155.

LYONS W. R., LI C. H., JOHNSON R. E., 1958. The hormonal control of mammary growth and lactation. Rec. Prog. Horm. Res., 14, 219-248.

MARTAL J., DJIANE J., 1977. Mammotrophin and growth promoting activities of placental hormone in sheep. J. Steroid Biochem., 8, 445-449. 
MARTEL P., HOUDEBINE L. M., 1982. Effect of various drugs affecting cytoskeleton and plasma membranes on the induction of DNA synthesis by insulin, epidermal growth factor and mammary explants. Biol. Cell, 44, 111-116.

MATUSIK R. J., ROSEN J. M., 1978. Prolactin induction of casein mRNA in organ culture. A model system for studying peptide hormone regulation of gene expression. J. biol. Chem., 253, 2343-2347.

Mc GRATH C. M., SOULE H. D., 1983. Renewal inhibition of human cell growth in vitro : cortisol and the recruitement of cells to terminal differenciation. J. cell Physiol., 116, 385-396.

Mc GRATH C. M., SOULE H. D., 1984. Calcium regulation of normal human mammary epithelial cell growth in culture. In vitro, 20, 652-662.

MEPHAM T. B., LAWRENCE S. E., PETERS A. R., HART I. C., 1984. Effects of exogenous growth hormone on mammary function in lactating goats. Horm. Metabol. Res., 16, 248-253.

MONAGHAN P., WARBURTON M. J., PERUSINGHE N., RUDLAND P. S., 1983. Topographical arrangement of basement membrane proteins in lactating rat mammary gland : comparison of the distribution of type IV collagen, laminin, fibronectin and Thy 1 at the ultrastructural level. Proc. nat. Acad. Sci. USA, 80, 3344-3348.

NAGARAH K., BOLANDER F. F., NICHOLAS K. R., TAKEMOTO T., TOPPER Y. J., 1981. Prolactin-induced accumulation of casein mRNA in mouse mammary explants : a selective role of glucocorticoid. Biochem. biophys. Res. Comm., 98, 380-387.

NAKHASI H. L., GRANTHAN F. H., GULLINO P. M., 1984, Expression of K-casein in normal and neoplastic rat mammary gland is under the control of prolactin. J. biol. Chem., 259, 14894-14898.

NIALL H. D., HOGAN M. L., SAUER R., ROSENBLUM I. Y., GREENWOOD F. C., 1971. Sequences of pituitary and placental lactogenic and growth hormone : evolution of primordial peptide by gene reduplication. Proc. nat. Acad. Sci. USA, 68, 866-869.

NICHOLAS K. R., SANKARAN L., TOPPER Y. J., 1981. The induction of $\alpha$-lactalbumin in rat mammary explants in the absence of exogenous prolactin : effects of progesterone and estrogen. Endocrinology, 109, 978-980.

NICHOLAS K. R., BOLANDER F. F., SANKARAN L., TOPPER Y. J., 1983. Deoxyribonucleic acid synthesis-dependent casein gene expression : species differences. Endocrinalogy, 112, 988-991.

NICHOLAS K. R., TOPPER Y. J., 1983. Anti-insulin receptor serum mimics the development role of insulin in mouse mammary explants. Biochem. biophys. Res. Comm., 111, 988-993.

NICOLL C. S., HEBERT N. J., RUSSELL S. M., 1985. Lactogenic hormones stimulate the liver to secrete a factor that acts synergistically with prolactin to promote growth of the pigeon crop-sac mucosal epithelium in vivo. Endocrinology, 116, 1449-1453.

OKA T., PERRY J. W., 1976. Studies on the function of glucocorticoid in mouse mammary epithelial cell differentiation in vitro. J. biol. Chem., 249, 7647-7652.

ONO M., OKA T., 1980a. The differential actions of cortisol on the accumulation of $\alpha$-lactalbumin and casein in mid-pregnant mouse mammary gland in culture. Cell, 19, 473-480.

ONO M., OKA T., 1980b. $\alpha$-lactalbumin-casein induction in virgin mouse mammary explants dose-dependent differential action of cortisol. Science, 207, 1367-1369.

ORMEROD E. J., RUDLAND P. S., 1982. Mammary gland morphogenesis in vitro: formation of branched tubules in collagen gels by a cloned rat mammary cell line. Dev. Biol., 91, 360-375.

OSSOWSKI L., BIEGEL D., REICH E., 1979. Mammary plasminogen activator : correlation with involution, hormonal modulation and comparison between normal and malignant tissue. Cell, 16. 929-940.

PARRY G., LEE E. Y. H., FARSON D., KOVAL M., BISSEL M. J., 1985. Collagenous substrate regulate the nature and distribution of glycosaminoglycans produced by differentiated cultures of mouse mammary epithelial cells. Exp. Cell Res., 156, 487-499.

PEEL C. J., BAUMAN D. E., GOREWIT R. C., SNIFFEN C. J., 1981. Effect of exogenous growth hormone on lactational performance in high yielding dairy cows. J. Nutr., 111, 1662-1671.

PORZIG E. F., STOCKDALE F. E., 1982. Mammary serum factor initiates cell growth in 3 T3 cell variants which are non-responsive to EGF. Exp. Cell Res., 41, 47-52. 
QASBA P. K., SAFAYA S. K., 1984. Similarity of the nucleotide sequences of rat $\alpha$-lactalbumin and chicken lysozyme genes. Nature, 308, 377-380.

RUDLAND P. S., BENNETT D. C., WARBURTON M. J., 1979. Hormonal control of growth and differentiation of cultured rat mammary gland epithelial cells. Cold Spring Harbor Conferences on Cell Proliferation, 6, 677-699.

RUDLAND P. S., PATERSON F. C., TWISTON-DAVIS A. C., WARBURTON M. J., 1983. Retinoicspecific induction of differentiation and reduction of the DNA synthesis rate and tumorforming ability of a stern cell line from a rat mammary tumor. J. nat. Canc. Inst., 70, 949-957.

RUDLAND P. S., TWISTON DAVIES A. C., TSAO S. W., 1984. Rat mammary preadipocytes in culture produce a trophic agent for mammary epithelia-prostaglandin E. J. cell. Physiol., 120, 364-376.

SALOMON D. S., LIOTTA L. A., KIDWELL W. R., 1981. Differential response to growth factor by rat mammary epithelium plated on different collagen substrata in serum-free medium. Proc. nat. Acad. Sci. USA, 78, 382.386.

SANKARAN L., QASBA P., TOPPER Y. J., 1984. Effects of estrogen-depletion on rat casein gene expression. Biochim. biophys. Res. Comm., 125, 682-689.

SANKARAN L., TOPPER Y. J., 1984. Prolactin-induced $\alpha$-lactalbumin activity in mammary explants from pregnant rabbits. Biochem. J., 217, 833-837.

SARKAR N. H., ETKIND P. R., LAFARGUES E. Y., WAITTINGTON E. S., 1984. Expression of an 86-kilodalton glycoprotein in an idiopathic mammary adenocarcinoma of a BALB/C mouse. Proc. nat. Acad. Sci. USA, 81, 4159-4163.

SERVELY J. L., 1983. Mécanisme d'action de la prolactine: Etude de la transmission de l'information hormonale dans la cellule épithéliale mammaire isolée. Thèse $3^{e} \mathrm{Cycle}$, Univ. Paris VI.

SERVELY J. L., N'GUEMA-EMANE M., HOUdEBINE L. M., DJIANE J., DELOUIS C., KELLY P. A., 1983. Comparative measurement of the lactogenic activity of ovine placental lactogen in rabbit and ewe mammary gland. Gen. comp. Endocr. 51, 255-262.

SHANNON J. M., PITELKA D. R., 1981. The influence of cell shape on the induction of functional differentiation in mouse mammary cells in vitro. In Vitro 17, 1016-1028.

SHING Y. W., KLAGSBURN M., 1984. Human and bovine milk contain different sites of growth factors. Endocrinology, 115, 273-282.

SINHA S. K., YUNIS A. A., 1983. Isolation of colony stimulating factor from human milk. Biochem. biophys. Res. Comm., 114, 797-803.

SMITH J. A., WINSLOW D. P., RUDLAND P. S., 1984. Different growth factors stimulate cell division of rat mammary epithelial, myoepithelial and stroma cell lines in culture. J. cell. Physiol., 119, 320-326.

SWANSON T. L., MOORE G. E., 1979. Bovine mammary gland contains a growth promoter for human breast cancer cell lines : colo 378 and colo 384 . Biochem. biophys. Res. Comm., 90, 1166-1171.

TAKETAMI Y., OKA T., 1983a. Tumor promoter 12-0-tetradecanoylphorbol 13-acetate like epidermal growth factor stimulates cell proliferation and inhibits differentiation of mouse mammary epithelial cells in culture. Proc. nat. Acad. Sci. USA, 80, 1646-1649.

TAKETAMI Y., OKA T., 1983b. Epidermal growth factor stimulates cell proliferation and inhibits functional differentiation of mouse mammary epithelial cells in culture. Endocrinology, 113. 871-877.

TAKETAMI Y., OKA T., 1983c. Biological action of epidermal growth factor and its functional receptors in normal mammary epithelial cells. Proc. nat. Acad. Sci. USA, 80, 2647-2650.

TALWALKER P. K., MEITES J., 1961. Mammary lobulo-alveolar growth induced by anterior pituitary hormones in adreno-ovariectomized and adreno-ovariectomised-hypophysectomized rats. Proc. Soc. exp. Biol. Med., 107, 882-883.

TERRADA N., OKA T., 1982. Selective stimulation of $\alpha$-lactalbumin synthesis and its mRNA accumulation by thyroid hormone in the differentiation of the mouse mammary gland in vitro. FEBS Letters, 149, 101-104.

TERRADA N., ONO M., NAGAMATSU Y., OKA T., 1982. The reversal of cortisol induced inhibition of $\alpha$-lactalbumin production by prostaglandins in the mouse mammary gland in culture. J. biol. Chem., 257, 1199-1202.

TERRADA N., LEIDERMAN L. J., OKA T., 1983. The interaction of cortisol and prostaglandins on 
the phenotypic expression of the $\alpha$-lactalbumin gene in the mouse mammary gland in culture. Biochem. biophys. Res. Comm., 111, 1059-1065.

TERRANOVA V. P., RAO C. N., KALEBIC T., MARGULIES I. M., LIOTTA L. A., 1983. Laminin receptor on human breast carcinoma cells. Proc. nat. Acad. Sci. USA, 80, 444-448.

TEYSSOT B., HOUDEBINE L. M., 1980. Role of prolactin in the transcription of $\beta$-casein and $28 \mathrm{~S}$ ribosomal genes in the rabbit mammary gland. Eur. J. Biochem., 110, 263-272.

TEYSSOT B., HOUDEBINE L. M., 1981. Role of progesterone and glucocorticoids in the transcription of the $\beta$-casein and $28 \mathrm{~S}$ ribosomal RNA genes in the rabbit mammary gland. Eur. J. Biochem., 114, 597-608.

TOMOOKA Y., IMAGAWA W., NANDI S., BERN H. A., 1983. Growth effect of lithium on mouse mammary epithelial cells in serum-free collagen gel culture. J. cell. Physiol., 117, 290-296.

VONDERHAAR B. K., 1977. Studies on the mechanism by which thyroid hormones enhance $\alpha$-lactalbumin activity explants from mouse mammary glands. Endocrinology, 100, 1423-1431.

VONDERHAAR B. K., SMITH G. H., PAULEY R. J., ROSEN J. M., TOPPER Y. J., 1978. A difference between mammary epithelial cells from mature virgin and primiparous mice. Cancer Res., 38, 4059-4065.

WASNER G., HENNERMANN I., KRATOCHWIL K., 1983. Ontogeny of mesenchymal androgen receptors in the embryonic mouse mammary gland. Endocrinology, 113, 1771-1778.

WICHA M. S., LIOTTA L. A., VONDERHAAR B. K., KIDWELL W. R., 1980. Effects of inhibition of basement membrane collagen deposition on rat mammary gland development. Develop. Biol., 80, 253-266.

WICHA M. S., LOWRIE G., KOHN E., BAGANDOSS P., MAHN T., 1982. Extracellular matrix promotes mammary epithelial growth and differentiation in vitro. Proc. nat. Acad. Sci. USA, 79. 3213-3217.

WILDE C. J., HASAN H. R., MAYER R. J., 1984. Comparison of collagen gels and mammary extracellular matrix as substrata for study of terminal differentiation in rabbit mammary epithelial cells. Exp. Cell Res., 151, 519-532.

YU-LEE L. Y., ROSEN J. M., 1983. The rat casein multigene family. Fine structure of the $\gamma$-casein gene. J. biol. Chem., 258, 10794-10804. 